\title{
Nucheal Located Epidermal Cyst: Successful Surgical Approach in a Bulgarian Patient
}

\author{
Valeri Malev ${ }^{1}$, Georgi Tchernev ${ }^{1,2 *}$ \\ ${ }^{1}$ Onkoderma-Clinic for Dermatology, Venereology and Dermatologic Surgery, General Skobelev 26, 1606 Sofia. \\ ${ }^{2}$ Medical Institute of Ministry of Interior (MVR-Sofia), Department of Dermatology, Venereology and Dermatologic Surgery Sofia, Bulgaria
}

Received: November 29, 2019; Accepted: November 29, 2019; Published: December 02, 2019

*Corresponding author: Prof Dr. Georgi Tchernev, Onkoderma-Clinic for Dermatology, Venereology and Dermatologic Surgery, General Skobelev 26, 1606 Sofia; 2Medical Institute of Ministry of Interior (MVR-Sofia), Department of Dermatology, Venereology and Dermatologic Surgery Sofia, Bulgaria. E-mail: georgi_tchernev@yahoo.de

Epidermal cysts are the most common cutaneous cysts and one of the common skin tumors [1]. Usually they appear on the face, scalp, neck, back and shoulders, but could also be seen on extremities, chest, scrotum, mucosa and inner organs [2,3]. They are benign tumors of dermis and hypodermis, arising from the hair follicle as a cystic proliferation of epidermal cells, forming cavity, filled with keratin and fat deposits [1]. The wall of the epidermal cyst is usually thin, built of squamous cells and a thick layer of granular cells, which resembles the epidermis itself [1]. Due to the thin wall the epidermal cysts often rupture, causing painful inflammation and infection [1].

In the following we present a 43 year old male with a big epidermal cyst on the neck. According to the patient it appeared 4 years ago, growing constantly since then. For several times it got inflamed, resulting in redness, pain and secretion of bright semi-liquid substance. In addition the patient has vitiligo on his hands since childhood.

On examination, we observed a $5 \mathrm{~cm}$ large, soft, poorly movable tumor formation on the neck, just on the border, dividing hair and neck. The lesion was hyperemic, painful with secretion of white substance from the central papilla. The lesion was removed surgically with elliptical excision with no evidence of postoperative neurological complications and head movement limitations [Figure. 1,2,3]. The histopathological diagnosis confirmed ruptured epidermal cyst with chronic granulomatous inflammation. The patient was followed-up for 2 months with no evidence of recurrence.

Epidermal cysts are congenital and acquired [4]. The congenital cysts are usually located on the midline of the body and arise from proliferation of entrapped ectodermal cells during the early embryogenesis [4]. Acquired cysts are located mainly in the head and neck region [5]. Implantation of epidermal cells into the deep mesenchymal tissues is induced by a variety of factors such as: local trauma, constant pressure on the skin, local inflammation of the hair follicle and even insect bite [5,6]. In the presented patient the most probable factors for forming of an epidermal cyst are the long-standing pressure and inflammation between the skin folds on the neck. Infection of eccrine channels with HPV can cause cysts formation on palms and soles, and multiple cysts on the body can be associated with Gardner syndrome $[7,8]$.
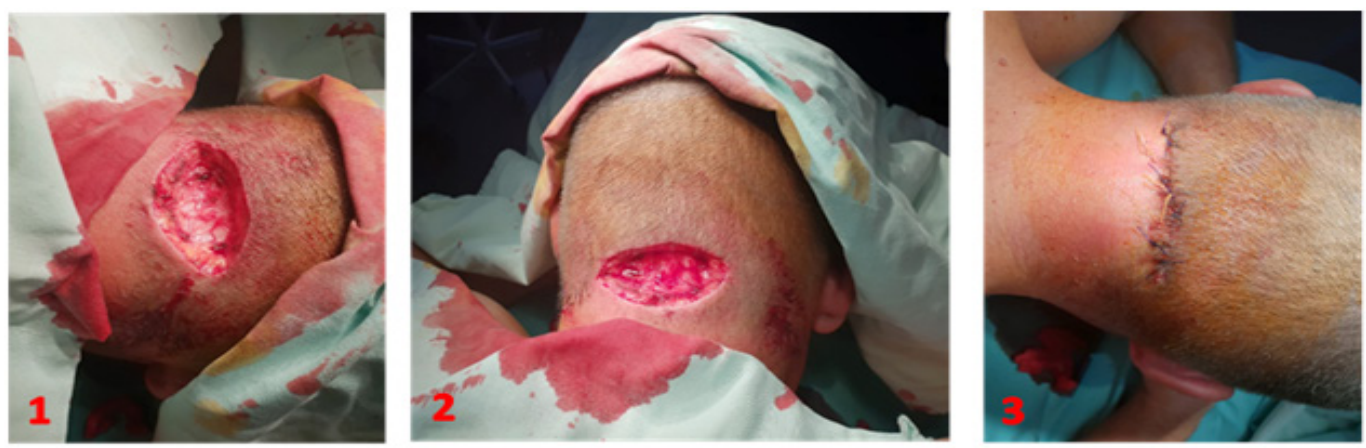

Figure: 1, 2 - Intraoperative look at a resected epidermal cyst on the neck of a 43 year old male.

Figure: 3 - Postoperative look at a closed operative wound with a classical, discontinued suture. 
Epidermal cysts (EC) are usually asymptomatic, but because of the thin wall, they often rupture, causing inflammation, pain and stiffness [5]. Although ECs are benign formation a study of Bauer et al. shows that 2,2\% of all ECs may develop into a well differentiated squamous cell carcinoma. Therefore all epidermal cysts must be evaluated histopathologically after surgical excision [9]. The clinical diagnosis could be supported by ultrasonography, fine needle aspiration cytology, CT and MRI [5]. The differential diagnosis includes: lipoma, neurofibroma, salivary gland adenoma, vascular lesion and fibroma [10].

The most common therapeutic option for epidermal cyst is the surgical excision [1] with removing of the whole cyst with no remaining parts of it, which reduces the risk of recurrence [1]. It is advisable to remove the cyst unruptured, in order to guarantee its integrity [1]. The classic elliptical excision is appropriate for the most localizations, limits the recurrence rate and provides a tension-free operative wound with good cosmetic results [1].

Mehrabi et al. studies the therapeutic results in 646 patients after punch excision of epidermal and pillar cysts [11]. As an advantage they highlight the easy and fast procedure, fast wound healing and good cosmetic results [11]. Recurrence rate varies between $3,6 \%$ and $8,3 \%$. The most common recurrence appears from cysts on the back $(13,8 \%)$ and ears $(13 \%)$ [11].

Another surgical approach is the endoscopic one. There the cut is hidden in the hair or behind the ears and a lesion on the visible part of the face is removed via endoscope [12]. Im JT et al. compare two groups of totally 275 patients after a direct or endoscopic excision of benign formation on the face, mainly the forehead (90\%) [12]. The results of this study shows that there is no statistically significant difference in the recurrence rate of the two approaches and in addition a better patient satisfaction in the group with endoscopic excision. Therefore in selected localizations and tumor sizes endoscopic resection can be also a valuable treatment option [12]. A good cosmetic result can also be achieved in localization on the cheeks, where an intraoral approach with careful dissection of musculus Masseter allows reaching and excision of the epidermal cyst without skin cut [10].

In the presented case we chose the classic elliptical excision because of the big size of the lesion, the local inflammation and relatively out of sight region. Besides that we needed to guarantee a tension free operative wound without head movement limitations.

\section{References}

1. Weir $\mathrm{CB}$ and Hilaire NJ. Epidermal Inclusion Cyst. InStatPearls [Internet].

2. Handa U, Kumar S and Mohan H. Aspiration cytology of epidermoid cyst of terminal phalanx. Diagnostic cytopathology. 2002; doi:10.1002/ dc. 10075

3. Sahoo MR, Gowda MS, and Behera SS. Unusual site and uncommon presentation of epidermoid cyst: a rare case report and review of literature. Case Reports. 2013;2013.doi:10.1136/bcr-2012-007907

4. Kandogan T, Koc M, Vardar E, Selek E and Sezgin O. Sublingual epidermoid cyst: a case report. J Med Case Reports. 2007; 1:87.
5. Rao A, Rao B, Kurian MJ and Pai RR. Two rare presentations of epidermal cyst. Journal of clinical and diagnostic research: JCDR. 2014; doi: 10.7860/JCDR/2014/8070.4982

6. Mahalakshmi S, Reddy S, Ramamurthy TK and Shilpa B. Rare locations of epidermoid cyst: case reports and review. Ethiopian journal of health sciences. 2016; doi: 10.4314/ejhs.v26i6.14

7. Weedon D. Weedon's skin pathology. Elsevier. 3rd ed. London: Elsevier 2010.

8. Pandya KA and Radke F. Benign skin lesions: lipomas, epidermal inclusion cysts, muscle and nerve biopsies. Surgical Clinics. 2009, 1;doi: 10.1016/j.suc.2009.03.002

9. Bauer BS and Lewis JV. Carcinoma arising in sebaceous and epidermoid cysts. Annals of plastic surgery. 1980; doi: 10.1097/00000637198009000-00008

10. Chen B, Lu H, Ren C, Ma L, Hu X, Qi H, et al. Excision of sebaceous cyst by intraoral approach: A case report. Medicine. 2017; doi: 10.1097/ MD.0000000000008803

11. Mehrabi D, Leonhardt JM and Brodell RT. Removal of keratinous and pilar cysts with the punch incision technique: analysis of surgical outcomes. Dermatologic surgery. 2002 ;28(8):673-677.

12. Im JT, Park BY, Lim SY, Pyon JK, Bang SI, Oh KS, et al. Endoscopic excision of benign facial tumors: a decade of experience with 152 patients. Annals of plastic surgery. 2014; doi: 10.1097/SAP.0b013e318268a8e5 